\title{
The $L_{\infty}$-algebra of the S-matrix
}

\section{Alex S. Arvanitakis}

The Blackett Laboratory, Imperial College London, Prince Consort Road London SW' 2AZ, U.K.

E-mail: a.arvanitakis@imperial.ac.uk

ABSTRACT: We point out that the one-particle-irreducible vacuum correlation functions of a QFT are the structure constants of an $L_{\infty}$-algebra, whose Jacobi identities hold whenever there are no local gauge anomalies. The LSZ prescription for S-matrix elements is identified as an instance of the "minimal model theorem" of $L_{\infty}$-algebras. This generalises the algebraic structure of closed string field theory to arbitrary QFTs with a mass gap and leads to recursion relations for amplitudes (albeit ones only immediately useful at tree-level, where they reduce to Berends-Giele-style relations as shown in [1]).

Keywords: BRST Quantization, Scattering Amplitudes, String Field Theory, Anomalies in Field and String Theories

ARXIV EPRINT: 1903.05643 


\section{Contents}

1 Introduction 1

$2 \quad L_{\infty}$-algebras $\quad 3$

2.1 The minimal model theorem for cyclic $L_{\infty}$-algebras 5

$\begin{array}{lll}3 & \text { The } L_{\infty} \text {-algebra of correlators } & 7\end{array}$

4 The $L_{\infty}$-algebra of the S-matrix 9

$\begin{array}{llr}5 & \text { Discussion } & 15\end{array}$

$\begin{array}{ll}\text { A Cyclic Hodge-Kodaira decompositions } & 18\end{array}$

B Path integral derivation of the Zinn-Justin equation from the quantum $\begin{array}{lr}\text { master equation } & 19\end{array}$

\section{Introduction}

Recall the setting of "textbook" quantum field theory (QFT) on Minkowski spacetime. The first goal therein is to calculate scattering amplitudes. These are obtained from correlation functions through the LSZ reduction formula [2]

$$
(i / \sqrt{Z})^{n} \int \prod_{i=1}^{n} d x_{i}\left[\exp \left(i x_{i} \cdot p_{i}\right)\left(\square_{x_{i}}+m^{2}\right)\left\langle\phi\left(x_{1}\right) \phi\left(x_{2}\right) \cdots \phi\left(x_{n}\right)\right\rangle_{\text {conn. }}\right]
$$

stated here for a connected S-matrix element (see [3] section 5-1-5) involving $n \geq 3$ outgoing spin-0 particles of the same species, mass $m^{2}>0$ and on-shell momenta $p_{1}, p_{2}, \ldots p_{n}$ respectively. $(\phi(x)$ is the field operator with nonzero amplitude $Z$ to create a 1-particle state while acting on the vacuum $|0\rangle,\left\langle\phi\left(x_{1}\right) \phi\left(x_{2}\right) \cdots \phi\left(x_{n}\right)\right\rangle_{\text {conn. }}$ is its connected $n$-point time-ordered vacuum correlation function, and $\left(\square_{x_{i}}+m^{2}\right)$ is the Klein-Gordon operator, acting on the correlator only.) One is then concerned with the calculation of correlators from first principles.

In this paper we make the following observations.

Firstly, that implicit in Zinn-Justin's 1974 proof of perturbative renormalisability of gauge theories [4] is an $L_{\infty}$-algebra (a generalisation of a Lie algebra involving brackets of any arity), whose structure constants are the one-particle-irreducible (1PI) correlators. We shall see that the $L_{\infty}$ Jacobi identities are equivalent to the Zinn-Justin equation, i.e. the classical Batalin-Vilkovisky (BV) [5-9] master equation for the 1PI generating functional $\Gamma[\Phi, \stackrel{\star}{\Phi}]$ (including sources $\stackrel{\star \star \star}{\Phi}$ for BRST transformations), which is in turn equivalent to the absence of anomalous terms in Slavnov-Taylor identities. We call this the $L_{\infty}$-algebra of correlators. 
Secondly, and less trivially, that the LSZ reduction formula endows the asymptotic 1-particle states with the structure of a minimal $L_{\infty}$-algebra (one without unary bracket), whose structure constants are the connected S-matrix elements. The $L_{\infty}$-algebraic interpretation is that this $L_{\infty}$-algebra of the $S$-matrix is a minimal model (no relation to the CFT notion) for the aforementioned $L_{\infty}$-algebra of 1PI correlators, and that the LSZ formula defines the corresponding quasi-isomorphism. We will explicitly show this for scalar theories with a mass gap only, but the argument directly generalises to any gapped theory.

The definition of $L_{\infty}$-algebras $[10,11]$ is now overdue. They generalise Lie algebras, so besides the structure constants $C_{b c}^{a}$ defining a binary bracket, one has $C_{b}^{a}, C_{b_{1} b_{2} b_{3}}^{a}, \ldots$ $C_{b_{1} \ldots b_{n}}^{a}, \ldots$ defining a unary, ternary, $\ldots n$-ary... bracket respectively, obeying symmetry identities and Jacobi identities (see (2.1)). Their first explicit appearance ${ }^{1}$ was in Zwiebach's work on closed bosonic string field theory [10] (CSFT): the genus-zero closed string correlators are the structure constants of an $L_{\infty}$-algebra, whose Jacobi identities imply gauge invariance of the string field lagrangian. Since then the concept has been picked up by mathematicians, who have articulated a general philosophy [17]: deformations of a structure (e.g. the complex structure of a complex manifold) are solutions of the MaurerCartan equation (2.12) (resp. the Kodaira-Spencer equation) of the associated $L_{\infty}$-algebra. For CSFT this is the string field equation of motion, whose solutions were argued to determine the conformal manifold of the worldsheet CFT [18]. For our $L_{\infty}$-algebra of correlators, this lore boils down to the best-known application of the 1PI functional $\Gamma$ in its guise as the Coleman-Weinberg effective potential [19]: extrema of $\Gamma$ determine vacuum states.

Minimal $L_{\infty}$-algebras have $C_{b}^{a}=0$. The relation between minimal $L_{\infty}$-algebras and S-matrices has been anticipated, originally in the context of $2 \mathrm{D}$ string theory by Witten and Zwiebach [20] and Verlinde [21], later for more general string (field) theories by authors including Kajiura [22], Münster and Sachs [23], and Konopka [24], and most recently for tree-level gravity and Yang-Mills by Nützi and Reiterer [25]. The novelty in our work is the generalisation beyond both string theory and perturbative expansions. To this end we write a proof of the minimal model theorem (that takes an $L_{\infty}$-algebra and gives the canonically associated minimal one), following a suggestion in [22], that mirrors the derivation by Jevicki and Lee [26] of the S-matrix generating functional from the 1PI functional $\Gamma$.

The recent resurgence of physics interest in $L_{\infty}$-algebras (e.g. [27-38]) mostly centres on gauge symmetries of classical theories. (Most relevant here is [28], which articulates the lore that classical BV master actions have canonical associated $L_{\infty}$-algebras $\left.[16,39-50]\right)$. Our observations suggest that $L_{\infty}$-algebraic approaches might be even more natural for quantum field theories: in a sense, $L_{\infty}$-algebras have been underlying QFT all along, as corroborated by (what we will argue is) the natural algebraic connection between vacuum correlators and Minkowski space S-matrix elements. We will suggest generalisations to other backgrounds and QFTs in the Discussion.

\footnotetext{
${ }^{1}$ They have appeared implicitly earlier in the D'Auria-Fré formulation of supergravity [12-14] and recognised as such in [15]; later they also appeared again implicitly in the work of [16] on higher-spin particles. (Both of these appearances predate the CSFT observation and I am grateful to Jim Stasheff for pointing this out.)
} 
Note added. The first version of this paper originally appeared on the arXiv at the same time as [1] which makes the same points (for tree-level theories) and in addition proves the relation between recursive amplitude formulae and the minimal model theorem. In particular, that is where the quasi-isomorphism appearing in the proof of the minimal model theorem for the $L_{\infty}$-algebra of correlators was originally given a physical interpretation as the collection of Berends-Giele "off-shell currents" [51] appearing in their namesake recursion relations.

\section{$2 \quad L_{\infty}$-algebras}

An $L_{\infty}$-algebra structure lives on a $\mathbb{Z}$-graded vector space $\mathcal{V}$. Denoting the $L_{\infty}$-algebra generators by $T_{a}$, each assumed to have some definite " $L_{\infty}$-degree" $\operatorname{deg} T_{a} \in \mathbb{Z}$, the symmetry and Jacobi identities are together encoded in the BRST-charge-like operator $Q$ (where $\partial / \partial z^{a}=\partial_{a}$ is a left derivative; our notation for symplectic supermanifolds is as in [38] appendix A)

$$
Q=\sum_{n=1}^{\infty} \frac{1}{n !} C_{b_{1} \ldots b_{n}}^{a} z^{b_{1}} \ldots z^{b_{n}} \frac{\partial}{\partial z^{a}}=Q^{a} \partial_{a}, \quad Q^{2}=0, \quad \operatorname{deg} Q=+1 .
$$

$Q$ acts on the space $C(\mathcal{V})$ of formal power series in variables $z^{a}$, with multiplication

$$
z^{a} z^{b}=(-1)^{\left(\operatorname{deg} z^{a}\right)\left(\operatorname{deg} z^{b}\right)} z^{b} z^{a}=(-1)^{a b} z^{b} z^{a}
$$

so $z^{a}$ is bosonic or fermionic according to $\operatorname{deg} z^{a} \in \mathbb{Z}$. The structure constants $C_{b_{1} \ldots b_{n}}^{a}$ (real or complex numbers) are defined so $Q$ increases this degree by 1 . The collection $\left\{z^{a}\right\}$ is the dual basis to the generators $T_{a}$ and the $z^{a}$ inherit their degrees from the $T_{a}$ : $\operatorname{deg} z^{a}=-\operatorname{deg} T_{a}$. A Lie algebra is the special case where all $\operatorname{deg} z^{a}=1 ; Q^{2}=0$ then reduces to the familiar Jacobi identity. The $L_{\infty}$ brackets are defined by

$$
\left[T_{b_{1}}, T_{b_{2}}, \ldots T_{b_{n}}\right]=C_{b_{1} \ldots b_{n}}^{a} T_{a}, \quad \operatorname{deg}\left[T_{b_{1}}, T_{b_{2}}, \ldots T_{b_{n}}\right]=1+\sum \operatorname{deg} T_{b_{i}}
$$

(This $L_{\infty}$ degree convention agrees with the "target space ghost number" of [10] and the "b-picture" of [27] after $\operatorname{deg} T_{a} \rightarrow-\operatorname{deg} T_{a}$.)

We exclusively use this geometric definition (called the "DGA-picture" in [28]) where $Q$ is interpreted as a vector field on a formal superspace $[39,52]$ with coordinates $z^{a}$, whose ring of "functions" is $C(\mathcal{V})$ by definition. A homomorphism $f$ of $L_{\infty}$-algebras $f: \mathcal{V} \rightarrow \mathcal{V}^{\prime}$ (henceforth morphism) is a degree 0 map of superspaces of the form (where each $f_{n} a_{1}^{\prime} a_{2} \ldots a_{n}$ is a constant)

$$
f^{\star}\left(z^{\prime}\right)^{a^{\prime}} \equiv f_{1 a}^{a^{\prime}} z^{a}+\frac{1}{2} f_{2} a_{1}^{a_{1} a_{2}} z^{a_{1}} z^{a_{2}}+\cdots \equiv \sum_{n=1}^{\infty} \frac{1}{n !} f_{n a_{1} a_{2} \ldots a_{n}} z^{a_{1}} z^{a_{2}} \cdots z^{a_{n}}
$$

which relates the vector fields by

$$
Q \circ f^{\star}=f^{\star} \circ Q^{\prime} .
$$


More precisely it is an $L_{\infty}$-degree-preserving linear map of the spaces of polynomials $f^{\star}$ : $C\left(\mathcal{V}^{\prime}\right) \rightarrow C(\mathcal{V})$ which respects multiplication $\left(f^{\star}(a b)=f^{\star}(a) f^{\star}(b)\right)$ and has zero constant part. It is an isomorphism if $f_{1}$ is invertible as a map $\mathcal{V} \rightarrow \mathcal{V}^{\prime}$.

An $L_{\infty}$-algebra with invariant inner product $\kappa$ is called cyclic. ${ }^{2}$ This is defined as a degree -1 symplectic form $\kappa=\kappa_{a b} d z^{a} d z^{b} / 2$ with constant coefficients $\kappa_{a b}$, annihilated by the Lie derivative $\mathcal{L}_{Q}$. The last condition is equivalent to

$$
C_{b_{1} \ldots b_{n+1}} \equiv \kappa_{a b_{1}} C_{b_{2} \ldots b_{n+1}}^{a}=(-1)^{b_{1} b_{2}} \kappa_{a b_{2}} C_{b_{1} \ldots b_{n+1}}^{a} .
$$

This notion agrees with the inner product on the space of string states of Zwiebach's [10] (cf. the "multilinear string functions" therein). $\kappa_{a b}$ has the symmetry

$$
\kappa_{a b}=\kappa_{b a},
$$

so the inverse $\kappa^{a b}$ is also symmetric. (The sign factor $(-1)^{(a+1)(b+1)}$ evaluates to +1 .)

Cyclic $L_{\infty}$-algebras are related to the BV formalism: the formal power series $\Theta$

$$
\Theta(z) \equiv \sum_{n=2}^{\infty} \frac{1}{n !} C_{b_{1} \ldots b_{n}} z^{b_{1}} \cdots z^{b_{n}}, \quad \operatorname{deg} \Theta=0
$$

defines a cyclic $L_{\infty}$-algebra with

$$
Q \equiv(\Theta,-)=\partial_{b} \Theta \kappa^{b a} \partial_{a}
$$

when $(\Theta, \Theta)=0$ (where $Q$ is the hamiltonian vector field of $\Theta$, and $(-,-)$ is the Poisson bracket of $\kappa)$, and vice versa. By introducing "antifields" $\stackrel{\star}{z}_{a}$ we can replace an arbitrary $L_{\infty}$-algebra $\mathcal{V}$ with a cyclic one $\mathcal{V}_{\text {cyclic }}$ (whose $\Theta$ is $(2.1)$ with $\partial_{a} \rightarrow \overleftarrow{z}_{a}$ ), yielding a surjective morphism $\mathcal{V}_{\text {cyclic }} \rightarrow \mathcal{V}$. (This is the "odd double" of [53].)

For completeness we mention the Maurer-Cartan equation for a cyclic $L_{\infty}$-algebra. Let $\Psi=\Psi^{a} T_{a}$ be a degree-zero element of the vector space $\mathcal{V}$ of a cyclic $L_{\infty}$-algebra (with coordinates $\Psi^{a}$ ). Consider the translation $z^{a} \rightarrow z^{a}+\Psi^{a}$ generated by the vector field $v_{\Psi} \equiv \Psi^{a} \partial_{a}$. Since the symplectic form $\kappa$ has constant coefficients,

$$
0=\exp \left(\mathcal{L}_{v_{\Psi}}\right)(\Theta(z), \Theta(z))=(\Theta(z+\Psi), \Theta(z+\Psi)),
$$

where $\Theta(z+\Psi) \equiv \exp \left(\mathcal{L}_{v_{\Psi}}\right) \Theta(z)$. Therefore, $\Theta(z+\Psi)$ is a formal power series in $z^{a}$ which will define a cyclic $L_{\infty}$-algebra iff the term linear in $z$ below vanishes:

$$
\Theta(z+\Psi)=\Theta(\Psi)+z^{a}\left(\frac{\partial}{\partial z^{a}} \Theta\right)_{z=\Psi}+O\left(z^{2}\right) .
$$

\footnotetext{
${ }^{2}$ The name is in reference to the $A_{\infty}$ generalisation: an $A_{\infty}$-algebra is defined exactly as above with the exception that the product $z^{a} z^{b}$ is only associative instead of graded commutative. Then a degree -1 symplectic form $\kappa$ is annihilated by the corresponding $Q$ iff the index-down structure constants have a cyclic symmetry under permutations. In terms of the relation between open string field theory and $A_{\infty}$ algebras, this corresponds to the fact that open string vertex operators are inserted on the $S^{1}$ boundary of the worldsheet.
} 
By degree-counting, this is true iff $\Psi$ solves the Maurer-Cartan equation

$$
\frac{\partial \Theta(\Psi)}{\partial \Psi^{a}}=0
$$

Note that there are convergence issues here because the $\Psi^{a}$ are reals (if the $L_{\infty}$-algebra is real) so $\Theta(\Psi)$ is an honest power series as opposed to a formal one.

Now consider the Jacobi identities $Q^{2}=0$ of an arbitrary $L_{\infty}$-algebra. These are organised by polynomial degree in $z^{a}$ : split $Q=Q_{0}+Q_{1}+Q_{2}+\ldots$ so each $Q_{n}$ increases polynomial degree by $n$, to obtain infinitely many identities

$$
Q_{0}^{2}=0, \quad Q_{0} Q_{1}+Q_{1} Q_{0}=0, \quad Q_{1}^{2}+Q_{2} Q_{0}+Q_{0} Q_{2}=0, \ldots
$$

The first gives $C_{b}^{a} C_{c}^{b}=0$; i.e. the $L_{\infty}$-algebra unary bracket $K$ (defined so $K\left(T_{a}\right)=C_{a}^{b} T_{b}$ ) has $K^{2}=0$. Since $K$ raises $L_{\infty}$-degree by $1, K$ is a cohomology operator on the graded vector space $\mathcal{V}$.

For a minimal $L_{\infty}$-algebra (i.e. $K=0$; in particular, Lie algebras are minimal as $L_{\infty}$ ones), the underlying vector space $\mathcal{V}$ is the cohomology of $K$. More generally, for any $L_{\infty}$-algebra one can put an $L_{\infty}$-algebra structure on the cohomology of $K$. This is called a minimal model for the original algebra, and all minimal models thusly obtained are isomorphic. For most purposes the study of an $L_{\infty}$-algebra can be reduced to that of its minimal model; for this reason, morphisms of $L_{\infty}$-algebras which correspond to isomorphisms of minimal models are particularly important and are known as quasi-isomorphisms. They are equivalently characterised as morphisms $f$ such that $f_{1}: \mathcal{V} \rightarrow \mathcal{V}^{\prime}$ is an isomorphism on the cohomologies of $K, K^{\prime}$ respectively.

\subsection{The minimal model theorem for cyclic $L_{\infty}$-algebras}

The minimal model theorem [54] claims a minimal $L_{\infty}$-algebra $\mathcal{V}_{\min }$ and an injective quasiisomorphism $\mathcal{V}_{\min } \rightarrow \mathcal{V}$. We here provide a short construction of a minimal model for a cyclic $L_{\infty}$-algebra $(\mathcal{V}, \Theta(z), \kappa)$ following a suggestion of Kajiura [22]: roughly, one extremises the hamiltonian $\Theta$, then backsubstitutes to find a hamiltonian for a minimal model. We will see later how this is exactly like the Jevicki-Lee prescription for the S-matrix [26].

Proof. (valid when $\left(\mathcal{V}^{\star}\right)^{\star} \cong \mathcal{V}$, e.g. in finite dimensions.)

We invoke a "cyclic Hodge-Kodaira decomposition" (see [22, 28, 55] and appendix A)

$$
\mathcal{V}=P \oplus P^{\perp}
$$

where $P$ is a subspace of $K$-cohomology representatives, $P^{\perp}$ is its $\kappa$-orthogonal complement, and $\kappa$ restricted to either is non-degenerate. We can find a partial inverse $G$ of $K$ on $P^{\perp}$ (a degree -1 map we will call the propagator), so $G K G=G, K G K=K, G^{2}=0$ and $G_{a c} \equiv \kappa_{a b} G_{c}^{b}=-(-1)^{a c} G_{c a}$. In particular $P^{\perp}=\operatorname{im}(K G) \oplus \operatorname{im}(G K)$ where each summand is $\kappa$-null. Abusing notation by calling $P$ both the subspace of $K$-cohomology representatives and a projector $P: \mathcal{V} \rightarrow \mathcal{V}$, we therefore have

$$
\kappa=\left(\begin{array}{cc}
\left.\kappa\right|_{P} & 0 \\
0 & \left.\kappa\right|_{P^{\perp}}
\end{array}\right),\left.\quad \kappa\right|_{\operatorname{im}(K G)}=\left.\kappa\right|_{\operatorname{im}(G K)}=0,\left.\quad \kappa\right|_{P}=P \kappa P \text { non-degenerate. }
$$


Now to construct a quasi-isomorphism $f: \mathcal{V}_{\min } \equiv P \rightarrow \mathcal{V}$. Let $\zeta^{a}$ be a basis of $P^{\star} \subset \mathcal{V}^{\star}$. Extend it to a basis $z^{a}$ of $\mathcal{V}^{\star}$ to write a direct sum

$$
z^{a}=\zeta^{a}+G_{b}^{a} C_{c}^{b} z^{c}+C_{b}^{a} G_{c}^{b} z^{c}, \quad P_{b}^{a} \zeta^{b}=\zeta^{a} .
$$

Here $P_{b}^{a}$ is the matrix of the projector $P$, and $C_{b}^{a}$ is the matrix of $K$. Specifying the last two terms as formal power series in $\zeta^{a}$ specifies the quasi-isomorphism (assuming both are $\left.O\left(\zeta^{2}\right)\right)$.

The candidate quasi-isomorphism $f: \mathcal{V}_{\text {min }} \rightarrow \mathcal{V}$ will be defined by the recursive formula (where $\bar{\Theta}$ is $\Theta$ without its quadratic part)

$$
f^{\star}\left(z^{a}\right)=\zeta^{a}-G^{a b} f^{\star}\left(\partial_{b} \bar{\Theta}\right)=\zeta^{a}-G_{b}^{a} f^{\star}\left(Q^{b}-Q_{0}^{b}\right) \quad\left(G^{a b} \equiv G_{c}^{a} \kappa^{b c}\right)
$$

Since $\partial \bar{\Theta}$ is $O\left(z^{2}\right)$, the right-hand side is defined as a formal power series in $\zeta$ (cf. (2.4)), and $f$ is an isomorphism in $K$-cohomology.

The sign in (2.17) is fixed by demanding (2.19) (important in the sequel):

$$
\begin{gathered}
C_{b}^{a} G_{c}^{b} Q^{c}=C_{b}^{a} G_{c}^{b} C_{d}^{c} z^{d}+C_{b}^{a} G^{b c} \partial_{c} \bar{\Theta}=C_{b}^{a} z^{b}+C_{b}^{a} G^{b c} \partial_{c} \bar{\Theta} \\
\Longrightarrow f^{\star}\left(C_{b}^{a} G_{c}^{b} Q^{c}\right)=0 .
\end{gathered}
$$

The last equality can be rewritten

$$
f^{\star}\left(G_{b}^{a} \partial_{a} \Theta\right)=0
$$

so we are in a sense "solving the equations of motion" derived from $\Theta(z)$. Then

$$
\Theta_{\min }(\zeta) \equiv f^{\star} \Theta(z), \quad\left(\kappa_{\min }\right)_{a b} \equiv P_{a}^{c} \kappa_{c d} P_{b}^{d}, \quad Q_{\min }^{a} \equiv \kappa^{a b} \frac{\partial \Theta_{\min }}{\partial \zeta^{b}}
$$

defines a minimal cyclic $L_{\infty}$-algebra if the master equation $\left(\Theta_{\min }, \Theta_{\min }\right)_{\min }=0$ is satisfied. A short calculation using $\partial \Theta_{\min } / \partial \zeta^{a}=P_{a}^{b} f^{\star}\left(\partial \Theta / \partial z^{b}\right)$ confirms

$$
\left(\Theta_{\min }, \Theta_{\min }\right)_{\min }=\kappa^{a b} P_{a}^{c} P_{b}^{d} f^{\star}\left(\partial_{c} \Theta \partial_{d} \Theta\right)=f^{\star}(\Theta, \Theta)=0
$$

where we used (2.20) and (2.15) in deriving the penultimate equality.

We have yet to show that $f$ is a morphism of $L_{\infty}$-algebras $\mathcal{V}_{\min } \rightarrow \mathcal{V}$. (This is not especially illuminating.) This is condition (2.5) which equivalently reads

$$
\left(f^{\star} \Theta, f^{\star} z^{a}\right)_{\min }=f^{\star}\left(\Theta, z^{a}\right) .
$$

Since $f$ is not invertible, it does not automatically give a morphism of (graded) Poisson brackets despite $f^{\star} \kappa=\kappa_{\text {min }}$. We now loosely follow Kajiura [22] and first replace $f$ with an invertible morphism $F: \mathcal{V} \rightarrow \mathcal{V}$, again recursively defined,

$$
F^{\star} z^{a}=\hat{z}^{a}-G_{b}^{a}\left(F^{\star} \bar{Q}^{b}\right), \quad\left(F^{-1}\right)^{\star} \hat{z}^{a}=z^{a}+G_{b}^{a} \bar{Q}^{b}, \quad\left(\bar{Q}^{a}=Q^{a}-Q_{0}^{a}\right)
$$

where $\hat{z}^{a}$ is $z^{a}$, renamed for clarity. $F$ preserves the symplectic form due to $\left.\kappa\right|_{\operatorname{im}(G K)}=0$ : $F^{\star} \kappa=\left(\kappa_{a b} d \hat{z}^{a} d \hat{z}^{b}\right) / 2$. It is therefore a symplectomorphism, which is a morphism of Poisson algebras, and thus both $F$ and $F^{-1}$ are $L_{\infty}$-isomorphisms. 
The transformed $L_{\infty}$-algebra structure is given by $\hat{Q}=F^{\star} \circ Q \circ\left(F^{-1}\right)^{\star}$. The point of this redefinition is that the new vector field, $\hat{Q}$, is tangent to the subspace $P$ of cohomology representatives except for its "non-minimal piece":

$$
\begin{aligned}
\hat{Q} & =Q^{a}\left(F^{\star} z\right) \frac{\partial\left(F^{-1}\right)^{\star} \hat{z}^{b}}{\partial z^{a}} \frac{\partial}{\partial \hat{z}^{b}} \\
& =F^{\star}\left(Q^{b}+G_{c}^{b} Q^{a} \partial_{a} \bar{Q}^{c}\right) \frac{\partial}{\partial \hat{z}^{b}} \\
& =F^{\star}\left(C_{c}^{b} z^{c}+\bar{Q}^{b}+G_{c}^{b} Q^{a} \partial_{a} \bar{Q}^{c}\right) \frac{\partial}{\partial \hat{z}^{b}} \\
& =\left(C_{c}^{b} \hat{z}^{c}\right) \frac{\partial}{\partial \hat{z}^{b}}+F^{\star}\left(-C_{c}^{b} G_{d}^{c} \bar{Q}^{d}+\bar{Q}^{b}+G_{c}^{b} Q^{a} \partial_{a} \bar{Q}^{c}\right) \frac{\partial}{\partial \hat{z}^{b}} \\
& =\left(C_{c}^{b} \hat{z}^{c}\right) \frac{\partial}{\partial \hat{z}^{b}}+F^{\star} \bar{Q}^{c} P_{c}^{b} \frac{\partial}{\partial \hat{z}^{b}} .
\end{aligned}
$$

(The Jacobi identities in the form $Q^{a} \partial_{a} Q^{b}=0$ were used for the last step.)

With this setup, we see that $\hat{f}: \mathcal{V}_{\text {min }} \rightarrow \mathcal{V}$ given by a straightforward projection to the cohomology, $\hat{f}^{\star} \hat{z}^{a}=P_{b}^{a} \hat{z}^{b} \equiv \zeta^{a}$, is an $L_{\infty}$-algebra morphism yielding a minimal model for $\hat{Q}$. Since $\hat{f}^{\star} \circ F^{\star}=f^{\star}, f^{\star}$ is an $L_{\infty}$-algebra morphism. This completes the proof.

The recursion (2.17) clearly terminates for the purposes of determining $f$ : a little counting shows that the $n$-th coefficient $f_{n b_{1} b_{2} \ldots b_{n}}^{a}$ (2.4) only depends on $C_{b_{1} \ldots b_{m}}^{a}$ for $m \leq n$ and $f_{m b_{1} b_{2} \ldots b_{n}}^{a}$ for $m \leq(n-1)$. It replaces the usual sum over trees in the proof of the minimal model theorem (e.g. Theorem 10.3.9 in [56]). This is highly suggestive of the combinatorics of the QFT 1PI generating functional, cf. also [22].

\section{The $L_{\infty}$-algebra of correlators}

Now consider a $D$-dimensional quantum field theory on a spacetime with coordinates $x^{\mu}$, with generating functional $Z[J]$ of correlation functions

$$
\delta^{n} Z /\left.\delta J\left(x_{1}\right) \ldots \delta J\left(x_{n}\right)\right|_{J=0}=\left\langle\phi\left(x_{1}\right) \phi\left(x_{2}\right) \ldots \phi\left(x_{n}\right)\right\rangle .
$$

Actually, for a gauge theory we consider the related functional $Z[J, \stackrel{\star}{\Phi}]$ where $\stackrel{\star \star}{\Phi}$ is a source for BRST transformations: differentiating $Z[J, \stackrel{\star}{\Phi}]$ with respect to $\stackrel{\star}{\Phi}$ leads to Ward identities for BRST symmetry. If a classical action is available, $Z[J, \stackrel{\star}{\Phi}]$ has a path integral expression

$$
Z[J, \stackrel{\star}{\Phi}]=\int \mathcal{D} \phi \exp \left(\frac{i}{\hbar} S[\phi, \stackrel{\star}{\Phi}+\delta \Psi / \delta \phi]+\int d x J(x) \phi(x)\right)
$$

where $S[\phi, \stackrel{\star}{\phi}]$ is the BV master action with bare fields $\phi$ (including ghosts) and antifields $\stackrel{\star}{\phi}$ and $\Psi$ is a gauge-fixing fermion. $S=S_{\mathrm{cl}}+O(\hbar)$ satisfies the quantum master equation,

$$
-2 i \hbar \Delta S+(S, S)=0, \quad \Delta S=(-1)^{\phi} \frac{\delta^{2} S}{\delta \phi(x) \delta^{\star} \dot{\phi}(x)}
$$

and its lowest order in $\hbar$ part $S_{\mathrm{cl}}$ solves the classical master equation. (The antibracket $(-,-)$ here is the usual expression $(\phi(x), \stackrel{\star}{\phi}(y))=\delta(x-y)$. The above is the usual setup of 
the BV formalism for arbitrary gauge theories, as reviewed comprehensively in e.g. [28, 57] and succinctly in e.g. [58]. We omitted Lorentz or other indices on $\phi$.

As an example of the above (from [59]) we briefly mention the Maxwell theory of a gauge potential $a_{\mu}(x)$ with field strength $f_{\mu \nu} \equiv 2 \partial_{[\mu} a_{\nu]}$ on 4-dimensional Minkowski space. Fields $\phi$ are $a_{\mu}$, a ghost $c$ of degree +1 , and in the non-minimal sector (only needed for gauge fixing) the Nakanishi-Lautrup field $b$ of degree 0 and an "antighost" $\bar{c}$ of degree -1 . Their antifields $\stackrel{\star}{\phi}$ are respectively ${ }_{a}^{\mu}, \stackrel{\star}{c}, \stackrel{\star}{c}, \stackrel{\star}{b}$ of degrees $-1,-2,0,-1$ respectively. The gauge-fixing fermion enforcing e.g. Lorenz gauge $\left(\partial^{\mu} a_{\mu}=0\right)$ is $\Psi=\int i \bar{c} \partial^{\mu} a_{\mu}$. Then

$$
S[\phi, \stackrel{\star}{\phi}]=\int d^{4} x-\frac{1}{4} f_{\mu \nu} f^{\mu \nu}+\stackrel{\star}{a}^{\mu} \partial_{\mu} c+i \stackrel{\star}{c} b
$$

solves (3.3). $Z[J, \stackrel{\star}{\Phi}]$ is a path integral with measure $\int \mathcal{D} a \mathcal{D} c \mathcal{D} b \mathcal{D} \bar{c}$ and we find in the integrand

$$
S[\phi, \stackrel{\star}{\Phi}+\delta \Psi / \delta \phi]=\int d^{4} x-\frac{1}{4} f_{\mu \nu} f^{\mu \nu}+\left(\stackrel{\star}{A}^{\mu}-i \partial^{\mu} \bar{c}\right) \partial_{\mu} c+\left(i \stackrel{\star}{\bar{C}}-\partial^{\mu} a_{\mu}\right) b .
$$

We thus see that $\stackrel{\star}{A}^{\mu}, \stackrel{\star}{C}$ are classical sources for the corresponding gauge fixed BRST transformations. (The other components of $\stackrel{\star \star \star}{\Phi}$ drop out in this simple example.)

The Legendre transform in the sense of formal power series [60] with respect to $J(x)$ of the connected generating functional $W=\log Z$ is the 1PI generating functional $\Gamma[\Phi, \stackrel{\star}{\Phi}]$ : define $\Phi(x)$ as a functional of $J$ and $\stackrel{\star}{\Phi}$ by

$$
\Phi(x)=\frac{\delta W[J, \stackrel{\star}{\Phi}]}{\delta J(x)}
$$

and invert it so $J$ is expressed using $\Phi, \stackrel{\star}{\Phi}$. Then

$$
\Gamma[\Phi, \stackrel{\star}{\Phi}]=-i \hbar\left(W-\int J(x) \Phi(x) d x\right),
$$

and $\delta \Gamma / \delta \Phi(x)=i \hbar(-1)^{J} J(x)$. Using this and integration by parts in the path integral,

$$
(\Gamma, \Gamma)=\left\langle\hbar^{-2}(-2 i \hbar \Delta S+(S, S))\right\rangle_{J[\Phi]} \equiv \int \mathcal{D} \phi \frac{1}{\hbar^{2} Z[J, \stackrel{\star}{\Phi}]} e^{\int J \phi}(-2 i \hbar \Delta S+(S, S)),
$$

where $J=J[\Phi],\langle\cdots\rangle_{J}$ is an expectation value in the presence of the source $J$ (see appendix B) and we defined an antibracket $(\Phi(x), \stackrel{\star}{\Phi}(y))=\delta(x-y)$. Therefore, if $S$ solves the quantum master equation (3.3), $\Gamma$ solves the classical master equation, and vice versa inside correlators, since $J$ is an arbitrary source (cf. the Schwinger-Dyson equations).

In this context the classical master equation $(\Gamma, \Gamma)=0$ for the 1PI functional is known as the Zinn-Justin equation [4]. It was originally found in the context of Yang-Mills, but can in fact be used to remove the divergences of fairly arbitrary field theories, as was done in [61] (see also the review [62]). Obstructions to the Zinn-Justin equation can be shown to correspond to local gauge anomalies; its validity implies Slavnov-Taylor identities. If the 1PI functional $\Gamma$ arises from a path integral involving a BV quantum master action $S$ 
as above, this is immediate from (3.8). (We refer to the review [57] sections 8.4 and 8.5 for more on this point and for a proof of (3.8).) We prefer however to think of $\Gamma$ as the fundamental object, not necessarily expressed as an $\hbar$-expansion or path integral.

We take $\Gamma$ along with the antibracket pairing between $\Phi$ and $\stackrel{\star}{\Phi}$ to define the cyclic $L_{\infty}$-algebra of correlators. Assuming $(\Gamma, \Gamma)=0$ we need only check that ${ }^{3}$

$$
\frac{\delta \Gamma}{\delta \Phi}=\frac{\delta \Gamma}{\delta \stackrel{\star}{\Phi}}=0 \quad \text { at } \Phi=\stackrel{\star}{\Phi}=0
$$

to match the form of (2.8); the structure constants of the $L_{\infty}$-algebra of correlators are then obtained by expanding $Q=(\Gamma,-)$ in $(\Phi, \stackrel{\star}{\Phi})$ around $\Phi=\stackrel{\star}{\Phi}=0$. These conditions have physical interpretations. $\delta \Gamma / \delta \Phi=0$ at $\Phi=0$ (which we need anyway for the Legendre transform) says that $\phi(x)$ has vanishing vacuum expectation value. This is usually violated in the context of spontaneous symmetry breaking, but can be remedied by a constant field shift for QFTs with Poincaré-invariant vacuum on Minkowski space. The other condition, $\delta \Gamma / \delta^{\star} \stackrel{\star}{\Phi}=0$, expresses the absence of BRST anomalies.

This setup applies of course to the closed bosonic string field theory of Zwiebach [10]. The "quantum string action" therein ( $S$ in our notation) is written as a genus expansion - equivalently, an $\hbar$-expansion - and satisfies the quantum master equation (3.3) as a consequence of the identities satisfied by the string products; in other words, as a consequence of the "loop $L_{\infty}$-algebra" [63] Jacobi identities (which at tree-level reduce to the well-known Jacobi identities of the ordinary $L_{\infty}$-algebra of closed string field theory). We have here translated these identities into the Zinn-Justin equation for the string field theory 1PI effective action $\Gamma$. The upshot is that the ordinary $L_{\infty}$-algebra structure defined by $\Gamma$ encodes both the well-known $L_{\infty}$-algebra of tree-level closed string field theory as well as the higher-genus contributions that deform it into a loop $L_{\infty}$-algebra; the price to be paid is that the $L_{\infty}$-algebra of $\Gamma$ is not defined over $\mathbb{C}$, but rather over $\mathbb{C}[\hbar]$ (complex formal power series in $\hbar$ ).

Before moving on we acknowledge that we have not actually specified the underlying vector space $\mathcal{V}$ on which the $L_{\infty}$ brackets act, so the construction in this section is so far formal. Fixing $\mathcal{V}$ at this level of generality is difficult. For the more concrete case of a scalar QFT on Minkowski space, we define $\mathcal{V}$ in the next section; it is simply a direct sum of on-shell wavefunctions (i.e. states appearing in the S-matrix) and Schwartz functions (i.e. functions of rapid decrease). One expects a similar picture for more general theories.

\section{The $L_{\infty}$-algebra of the S-matrix}

Our claim here is that the LSZ reduction formula can be interpreted as the quasiisomorphism appearing in the above proof of the minimal model theorem for cyclic $L_{\infty^{-}}$ algebras. To this end we recall and clarify the observation originally due to Jevicki and

\footnotetext{
${ }^{3}$ When this fails, but $(\Gamma, \Gamma)=0$ holds, one gets what should be called a "cyclic curved $L_{\infty}$-algebra". This is discussed already in [10] (albeit without this name, which appeared later) in the context of closed bosonic string field theory, where this failure is related to the failure of the string background to be conformal. As it is not clear whether one can say anything interesting without shifting the background back to a conformal one, we choose not to elaborate in this case.
} 
Lee $[26]$ (see also $[64,65]$ and the textbooks $[66,67]$ ) that the S-matrix functional [68] is obtained from the 1PI functional $\Gamma[\Phi, \stackrel{\star}{\Phi}]$ by extremising it.

We assume here the same setup as in the scalar LSZ formula (1.1). Our QFT only has one real scalar field operator $\phi(x)$ with 2-point function (propagator)

$$
G(x-y) \equiv\langle\phi(x) \phi(y)\rangle=\int_{0}^{\infty} d\left(\mu^{2}\right) \rho\left(\mu^{2}\right) G_{F}\left(x-y ; \mu^{2}\right)
$$

where $G_{F}\left(x ; \mu^{2}\right)$ is the usual Feynman propagator for the mass $\mu^{2}$ Klein-Gordon equation, satisfying $\left(\square+\mu^{2}\right) G_{F}\left(x ; \mu^{2}\right)=-i \hbar \delta(x)$. This is the Källén-Lehmann spectral representation $[69,70]$. The spectral function $\rho\left(\mu^{2}\right)$ takes the form

$$
\rho\left(\mu^{2}\right)=Z \delta\left(m^{2}-\mu^{2}\right)+\sigma\left(\mu^{2}\right), \quad 0<Z<1
$$

appropriate for a scalar QFT whose asymptotic free particle states have mass $m^{2}$, so the smooth function $\sigma\left(\mu^{2}\right)$ is only non-zero above a threshold $m_{\text {thresh }}^{2}>m^{2}$ for production of multiparticle states. We assume furthermore that there are no asymptotic states besides the ones created by $\phi(x)$, and suggest a remedy in the Discussion.

These assumptions are fairly restrictive, but our arguments should apply mutatis mutandis to any theory where states entering the S-matrix are all massive. A considerable technical simplification for scalar theories is that we need not introduce gauge symmetry, so we can without loss of generality let $\Gamma$ and $W$ be independent of $\stackrel{\star}{\Phi}$. Therefore, the Zinn-Justin equation $(\Gamma, \Gamma)=0$ is trivially satisfied.

The LSZ formula (1.1) relates the connected S-matrix generating functional $\mathcal{A}[\varphi]$ to $W[J]$ by evaluation of the latter on the source $J_{\varphi}$, defined by

$$
\int d x J_{\varphi}(x) f(x)=\frac{i}{\sqrt{Z}} \int d x \varphi(x)\left(\square_{x}+m^{2}\right) f(x)
$$

for functions $f$ such that the right-hand side makes sense. Here $\varphi(x)$ is the wavefunction of an asymptotic 1-particle state, i.e. an S-matrix state:

$$
\left(\square_{x}+m^{2}\right) \varphi(x) \equiv\left(\partial_{t}^{2}-\nabla^{2}+m^{2}\right) \varphi(x)=0,
$$

so the integrand above is in fact a total derivative, and $J_{\varphi}$ is a somewhat bizarre distribution supported at infinity (more on this later). A subtlety here is that $\mathcal{A}[\varphi]$ has a non-zero universal quadratic part associated to trivial $1 \rightarrow 1$ scattering, while the quadratic in $\varphi$ part of $W\left[J_{\varphi}\right]$ vanishes for our gapped scalar QFT using (4.1) and (4.2) (see [3] section 51-5). Thus, $W\left[J_{\varphi}\right]$ is the generating functional of non-trivial connected S-matrix elements, and we will write $\mathcal{A}[\varphi]=W\left[J_{\varphi}\right]$, implicitly discarding the quadratic part.

To rewrite this in terms of the $1 \mathrm{PI}$ functional, we need to solve $\delta \Gamma / \delta \Phi(x)=i \hbar J(x)$ for $\Phi$ in terms of $J$. This is the (inverse) Legendre transform of formal power series [60]. Since $G(x)$ of (4.1) is the inverse of $\delta^{2} \Gamma /\left.\delta \Phi^{2}\right|_{\Phi=0}$ up to prefactors, if $\bar{\Gamma}$ is $\Gamma$ without its quadratic part, we find the general solution

$$
\Phi(x)=\int d y G(x-y)\left(J(y)+\frac{i}{\hbar} \frac{\delta \bar{\Gamma}}{\delta \Phi(y)}\right) .
$$


For $J=J_{\varphi}$, definition (4.3) and (4.1), (4.2), (4.4) give

$$
\int d y G(x-y) J_{\varphi}(y)=\hbar \sqrt{Z} \varphi(x)
$$

here crucially the multiparticle contribution $\sigma\left(\mu^{2}\right)$ to the spectral function (4.2) drops out, effectively replacing $G(x)$ by the (free) propagator $G_{F}\left(x ; m^{2}\right)$ up to a factor of $Z$. If $J_{\varphi}$ is interpreted as a "source at infinity" for an incoming/outgoing on-shell state with wavefunction $\varphi(x)$, this calculation states that the multiparticle states fail to contribute to the time evolution of $\varphi$ from $x^{0}= \pm \infty$ to finite values. The mass gap $m_{\text {thresh }}^{2}>m^{2}$ of (4.2) is crucial to this calculation.

We therefore find the following recursive formula defining $\Phi$ as a formal power series in the wavefunction $\varphi$ (cf. (2.17))

$$
\Phi_{\varphi}(x)=\hbar \sqrt{Z} \varphi(x)+\frac{i}{\hbar} \int d y G(x-y) \frac{\delta \bar{\Gamma}}{\delta \Phi(y)}
$$

which leads to the following formula for the S-matrix functional

$$
\mathcal{A}[\varphi]=i \hbar^{-1} \Gamma\left[\Phi_{\varphi}\right]+\int J_{\varphi}(x) \Phi_{\varphi}(x) d x .
$$

So far we have never dropped any boundary terms. We would like to now drop the second term in $\mathcal{A}[\varphi]$ above, which is proportional to the total derivative

$$
\int d x \varphi(x)\left(\square+m^{2}\right) \Phi_{\varphi}(x)=\int d x \partial_{\mu}\left(\Phi_{\varphi}(x) \partial^{\mu} \varphi(x)-\varphi(x) \partial^{\mu} \Phi_{\varphi}(x)\right)
$$

whenever $\varphi(x)$ solves the Klein-Gordon equation (4.4). This is anyway zero to order $O\left(\varphi^{2}\right)$. Obviously we cannot in general prove this vanishes (e.g. in the sense of formal power series) without estimates on 1PI correlators and without specifying the space of $\varphi(x)$. For $\varphi(x)$ an appropriate space is the space $V_{\mathrm{rwp}}$ of regular wave packets, i.e. of smooth solutions to the Klein-Gordon equation (4.4) with initial data of compact support in momentum space:

$$
\varphi(x)=\int \frac{d^{D-1} k}{\sqrt{(2 \pi)^{D-1} 2 E_{\vec{k}}}}\left(\alpha(\vec{k}) e^{i\left(E_{\vec{k}} t+\vec{k} \cdot \vec{x}\right)}+\bar{\alpha}(\vec{k}) e^{-i\left(E_{\vec{k}} t+\vec{k} \cdot \vec{x}\right)}\right), \quad E_{\vec{k}}=\sqrt{|\vec{k}|^{2}+m^{2}}
$$

(where $\alpha(\vec{k})$ is basically the spatial Fourier transform of the initial data $\left.\varphi\right|_{x^{0}=t=0}$ for $\varphi(x)$, $\bar{\alpha}(\vec{k})$ is its complex conjugate, and both are of compact support by assumption. Regular wave packets can approximate momentum eigenstates arbitrarily well, which is the primary consideration here.) These always vanish at infinity (Theorem XI.17 of [71]), so assuming that $\Phi_{\varphi}(x)$ does not blow up at infinity, (4.9) should not contribute to the S-matrix. This is also argued to be the case in $[26,65]$. As a final justification, we note that with the choice of $\mathcal{V}$ specified later in this section it is clear this total derivative term vanishes.

The upshot is the following formula for the S-matrix functional involving the 1PI functional alone [26] (also [64, 65]):

$$
\mathcal{A}[\varphi]=\frac{i}{\hbar} \Gamma\left[\Phi_{\varphi}\right] .
$$


One interpretation is that one obtains the S-matrix by evaluating $\Gamma[\Phi]$ on the solution of $\delta \Gamma / \delta \Phi(x)=0$ : writing

$$
\Gamma[\Phi]=\frac{1}{2}(i \hbar) \int d x \int d y K(x-y) \Phi(x) \Phi(y)+O\left(\Phi^{3}\right),
$$

we find (the $i \hbar$ right above absorbs superfluous factors below)

$$
\int d z K(x-z) G(z-y)=\delta(x-y) .
$$

Using this, (4.6) yields

$$
\int d y K(x-y) \varphi(y)=(\hbar \sqrt{Z})^{-1} J_{\varphi}(x)
$$

The right-hand side vanishes when integrated against any $f(x)$ of compact support by (4.3), so in some sense $\varphi(x)$, which is annihilated by the Klein-Gordon operator (4.4), is also annihilated by the operator $K$ derived from the 1PI effective action. (This is a check that $\varphi$ satisfies the Klein-Gordon equation with renormalised mass, as it anyway must if it is the wavefunction of a scattering state.) Of course, the right-hand side of (4.14) is very much non-zero. This apparent tension is resolved by interpreting $J_{\varphi}(x)$ as a change of boundary conditions: the equation $K f(x)=J_{\varphi}(x)+J(x)$ for $f(x)$ is interpreted as $K(g(x))=J(x)$ with $g(x)=f(x)-\hbar \sqrt{Z} \varphi(x)$ lying in $V_{\text {Schwartz }}$ i.e. the space of functions of rapid decrease; the point here being that $K$ is invertible when restricted to $V_{\text {Schwartz }} \cdot{ }^{4}$ Analogously, we see that $\Phi_{\varphi}(x)$ of (4.7) is the unique solution of

$$
\frac{\delta \Gamma}{\delta \Phi(x)}=i \hbar \int d y(K(x-y) \Phi(y))+\frac{\delta \bar{\Gamma}}{\delta \Phi(x)}=0
$$

if we assume $\Phi(x)-\hbar \sqrt{Z} \in V_{\text {Schwartz }}$. At tree level, this reduces to the well-known recipe for tree-level S-matrix elements from the classical action, recently exploited in e.g. [73, 74].

The argument above is clarified by considering the free theory: in that case $Z=1, K$ is proportional to $\left(\square+m^{2}\right)$, and $G$ is the Feynman propagator. The choice of Feynman boundary conditions for the propagator is related to the choice of boundary conditions in the path integral, which are in turn fixed by our decision to calculate vacuum correlators.

We now articulate the $L_{\infty}$-algebraic interpretation. We define the hamiltonian $\Theta[\Phi]$ and antibracket defining the $L_{\infty}$-algebra of correlators as

$$
\Theta[\Phi]=-\frac{i}{\hbar} \Gamma[\Phi], \quad(\Phi(x), \stackrel{\star}{\Phi}(y))=\delta(x-y), \quad \operatorname{deg} \Phi(x)=0, \quad \operatorname{deg} \stackrel{\star}{\Phi}(x)=-1 .
$$

$\Phi(x)$ and $\stackrel{\star}{\Phi}(x)$ play the role $z^{a}$ did in the general discussion previously. In particular $\Phi(x)$, $\stackrel{\star}{\Phi}(x)$ are not the values of fixed functions at some spacetime point $x$, but rather they are

${ }^{4}$ This is easiest to see from the Källén-Lehmann expression for the exact propagator $G(4.1)$ and (4.2), which should really be interpreted in momentum space, where $G$ is a multiplication operator. Assuming $G$ exists in this context as a multiplication operator on $V_{\text {Schwartz }}$, doing a Wick rotation shows it has an inverse, which is $K$ by definition. The position space expressions used here are then obtained by Fourier transform, which is anyway an isomorphism on $V_{\text {Schwartz. }}$. We refer to [72] chapter 14 for the relevant analysis background. 
linear functionals mapping functions $f: \mathbb{R}^{D} \rightarrow \mathbb{R}$ to their values at a spacetime point $x$ (much like $z^{a} \in \mathcal{V}^{\star}$ is a linear map $\mathcal{V} \rightarrow \mathbb{R}$. $)^{5}$ The various $L_{\infty}$-algebra brackets act on the space $\mathcal{V}$ spanned by their duals. Since $\operatorname{deg} \Phi=0, \operatorname{deg} \stackrel{\star}{\Phi}=-1, \mathcal{V}$ is concentrated in degrees 0 and +1 respectively. At degree 0 we have ordinary scalar fields $\phi(x)$ while at degree 1 we have degree-shifted scalars we will write $c \phi(x)$ using a degree +1 formal constant $c$.

Since $Q=\int d x(\Theta, \stackrel{\star}{\Phi}(x)) \delta / \delta \stackrel{\star}{\Phi}(x)$, the $L_{\infty}$-algebra structure constants can be read off the expansion in $\Phi(x)$ of

$$
(\Theta, \stackrel{\star}{\Phi}(x))=\int d y K(x-y) \Phi(y)+O\left(\Phi^{2}\right) .
$$

In particular the unary bracket $K$ reads

$$
K \phi(x)=c \int d y K(x-y) \phi(y), \quad K(c \phi(x))=0,
$$

and the 1PI $n$-point correlators $n \geq 3$ similarly define the $(n-1)$-ary bracket $\left[\phi_{1}, \phi_{2}, \ldots \phi_{n}\right](x)$. (Any bracket any of whose arguments involves $c$ vanishes. All the Jacobi identities are thereby satisfied.)

Therefore the vector space of the $L_{\infty}$-algebra of correlators is

$$
\mathcal{V}=V \oplus V[+1], \quad V \stackrel{K}{\rightarrow} V[+1]
$$

where $V$ is the following space of scalar fields $\phi(x)$

$$
\phi(x)=\varphi(x)+\phi_{\mathrm{S}}(x), \quad \text { i.e. } V=V_{\mathrm{rwp}} \oplus V_{\text {Schwartz }}
$$

a direct sum of regular wave packets $\varphi(x)$ (i.e. wavefunctions of asymptotic 1-particle states) along with $\phi_{\mathrm{S}}(x) \in V_{\text {Schwartz }}$ lying in the Schwartz space $V_{\text {Schwartz }}$ of rapidly-decreasing functions.

With this choice, the cohomology of $K$ at degree 0 is the regular wave packets:

$$
K \phi(x)=0 \Longleftrightarrow \int d y K(x-y)\left(\varphi(y)+\phi_{\mathrm{S}}(y)\right)=0 \Longleftrightarrow \phi_{\mathrm{S}}(x)=0 .
$$

(There is no tension with (4.14) here since $J_{\varphi}(x)$ is not an element of $\mathcal{V}$; it has been projected out. Hopefully our use of $K$ in both places to denote slightly different operators is not confusing.) Since $\phi_{\mathrm{S}}(x)=K G \phi_{\mathrm{S}}(x)$ in slightly abusive notation it is clear that a space of cohomology representatives at degree 1 is again the space of regular wave packets. Therefore, we find the underlying vector space of the putative minimal $L_{\infty}$-algebra

$$
\mathcal{V}_{\min }=V_{\mathrm{rwp}} \oplus V_{\mathrm{rwp}}[+1] .
$$

\footnotetext{
${ }^{5}$ This is apparently called the "fundamental confusion of calculus" and is elaborated on in [28] in this context. It is perhaps clearer to explain this way: since $z^{a}$ are a basis of $\mathcal{V}^{\star}$, if $v=v^{a} T_{a}$ with $v^{a} \in \mathbb{R}$ and $T_{a} \in \mathcal{V}$ are a basis, the map $\mathcal{V} \rightarrow \mathbb{R}$ defined by $v \rightarrow v^{a}$ (for any specific choice of index $a$ ) is simply $\left\langle z^{a} \mid v\right\rangle=$ $\left\langle z^{a} \mid T_{b}\right\rangle v^{b}=\delta_{b}^{a} v^{b}=v^{a}$. The confusion is that $\operatorname{deg} v^{a}=0$ (since $v^{a} \in \mathbb{R}$ is a number) but $\operatorname{deg} z^{a}=-\operatorname{deg} T_{a}$ (which is anyway necessary for $\left\langle z^{a} \mid T_{b}\right\rangle=\delta_{b}^{a}$ since numbers are in degree zero). With regard to determining the brackets on $\mathcal{V}$ from $Q$ acting on polynomials in $z^{a}$, we have e.g. $Q z^{a}=C_{b}^{a} z^{b}+O\left(z^{2}\right)$ (corresponds to (4.17)), $K v=K T_{b} v^{b}=\left(C_{b}^{a} v^{b}\right) T_{a} \Longrightarrow v^{a} \rightarrow C_{b}^{a} v^{b}$ (corresponds to (4.18)).
} 
We have been coy about discussing the cyclic inner product on the $L_{\infty}$-algebra of correlators (or that of the S-matrix) so far. The reason is that our sins - in recklessly taking duals of infinite-dimensional spaces - have now caught up with us. The canonical antibracket $(\Phi(x), \stackrel{\star}{\Phi}(y))$ of (4.16) formally defines the degree -1 symplectic form $\kappa=$ $\int d x \delta \Phi(x) \delta \stackrel{\star}{\Phi}(x)$ which is a pairing $V \times V[+1] \rightarrow \mathbb{R}$. A near-identical construction appears in Costello's treatment of $\phi^{4}$ theory (Example 5.1 of [75]), the difference being that he works on a compact Euclidean spacetime, where this $\kappa$ is well-defined.

We however must work on Minkowski spacetime in order to treat the on-shell external states. The above "naïve" bilinear form $\kappa$ blows up for regular wave packets $\varphi(x)$ : indeed from (4.10) we calculate

$$
\int d x \varphi_{1}(x) \varphi_{2}(x)=\int d t \int \frac{d^{D-1} k}{E_{\vec{k}}} \operatorname{Re}\left[\alpha_{1}(\vec{k}) \bar{\alpha}_{2}(\vec{k})+\alpha_{1}(\vec{k}) \alpha_{2}(\overrightarrow{-k}) e^{i\left(2 E_{\vec{k}}\right) t}\right] .
$$

The first term blows up due to a factor $\int d t 1$, while the second is singular due to the oscillatory integral $\int d t \exp \left(i 2 E_{\vec{k}}\right) t \propto \delta\left(2 E_{\vec{k}}\right)$ which should in some sense vanish since $E_{\vec{k}}$ is bounded away from zero by $m>0$. This requires regularisation. We thus smear $\varphi(x)$ around the mass-shell in momentum space by replacing

$$
\varphi(x)=\int d k_{0} \frac{d^{D-1} k}{\sqrt{(2 \pi)^{D-1} 2 E_{\vec{k}}}} \delta\left(k_{0}-E_{\vec{k}}\right)\left(\alpha(\vec{k}) e^{i k x}+\bar{\alpha}(\vec{k}) e^{-i k x}\right)
$$

with

$$
\varphi_{\varepsilon}(x)=\int d k_{0} \frac{d^{D-1} k}{\sqrt{(2 \pi)^{D-1} 2 E_{\vec{k}}}} \delta_{\varepsilon}\left(k_{0}-E_{\vec{k}}\right)\left(\alpha(\vec{k}) e^{i k x}+\bar{\alpha}(\vec{k}) e^{-i k x}\right)
$$

where $\delta_{\varepsilon}\left(k_{0}\right)$ is an e.g. Gaussian approximation of the Dirac delta:

$$
\delta_{\varepsilon}\left(k_{0}\right)=\frac{1}{2 \pi} \int d t \exp \left(i k_{0} t-\varepsilon t^{2}\right)=\frac{1}{\sqrt{4 \pi \varepsilon}} e^{-\left(k_{0}\right)^{2} / 4 \varepsilon}, \quad \varepsilon>0 .
$$

This can be seen as an $i \varepsilon$-prescription. Then $\int d x \varphi_{1, \varepsilon}(x) \varphi_{2, \varepsilon}(x)$ reads

$$
\sqrt{\frac{\pi}{2}} \int \frac{d^{D-1} k}{E_{\vec{k}}} \operatorname{Re}\left[\alpha_{1}(\vec{k}) \bar{\alpha}_{2}(\vec{k}) \varepsilon^{-1 / 2}+\alpha_{1}(\vec{k}) \alpha_{2}(\overrightarrow{-k}) \varepsilon^{-1 / 2} e^{-E_{\vec{k}}^{2} /(2 \varepsilon)}\right]
$$

where the first term diverges like $\varepsilon^{-1 / 2}$ as $\varepsilon \rightarrow 0$, but the second term vanishes.

We therefore define a regularised cyclic inner product $\kappa$ in the $L_{\infty}$-algebra of correlators by rescaling $\varphi(x)$ in $(4.20)$ by $(2 \varepsilon / \pi)^{1 / 4}$ :

$$
\begin{aligned}
\kappa\left(\phi_{1}, c \phi_{2}\right) & =\lim _{\varepsilon \rightarrow 0} \int d x\left(\left(\frac{2 \varepsilon}{\pi}\right)^{1 / 4} \varphi_{1, \varepsilon}(x)+\phi_{1, \mathrm{~S}}(x)\right)\left(\left(\frac{2 \varepsilon}{\pi}\right)^{1 / 4} \varphi_{2, \varepsilon}(x)+\phi_{2, \mathrm{~S}}(x)\right) \\
& =\int \frac{d^{D-1} k}{E_{\vec{k}}} \operatorname{Re}\left[\alpha_{1}(\vec{k}) \bar{\alpha}_{2}(\vec{k})\right]+\int d x \phi_{1, \mathrm{~S}}(x) \phi_{2, \mathrm{~S}}(x) .
\end{aligned}
$$

(Recall $\mathcal{V}=V \oplus V[1]$ where we write $V[1]$ with the degree +1 formal constant $c$.) Not only did we absorb the divergence - yielding a positive-definite inner product on $V_{\text {rwp }}$ - 
but we also rendered $V_{\text {rwp }} \kappa$-orthogonal to the Schwartz functions $\phi_{\mathrm{S}}(x)$. This realises a Hodge-Kodaira decomposition

$$
\mathcal{V}=P \oplus P^{\perp}, \quad P=\mathcal{V}_{\text {min }}=V_{\text {rwp }} \oplus V_{\text {rwp }}[+1], \quad P^{\perp}=V_{\text {Schwartz }} \oplus V_{\text {Schwartz }}[+1]
$$

in the sense of the proof of the minimal model theorem in the preceding section, for which the degree -1 map $G: \mathcal{V} \rightarrow \mathcal{V}$ defined by (where again $G(x-y)$ is (4.1))

$$
G: V[+1] \rightarrow V, \quad G(c \varphi(x))=0, \quad G\left(c \phi_{\mathrm{S}}(x)\right)=\int d y G(y-x) \phi_{\mathrm{S}}(y)
$$

and vanishing otherwise, is a "propagator" in that same sense.

We are not quite done yet however, as there are outstanding analytic issues in the presence of interactions (i.e. non-vanishing binary and higher $L_{\infty}$-brackets), as was originally pointed out in [1]. These even appear at tree level, where $\Gamma$ is the classical action: in the presence of an e.g. $\phi^{3}$ interaction, $\Gamma$ fails to be defined because the integral $\int d x \phi^{3}(x)$ blows up when $\phi \in V_{\text {rwp }}$. This is remedied in [1] by (effectively) inserting a position-dependent Gaussian in the $\phi^{3}$ term, at the price of Lorentz invariance. In the general case, beyond tree level, we regard these issues as part of the definition of $\Gamma$.

The upshot is that we have now realised the setting of the proof of the minimal model theorem as given in the preceding section for cyclic $L_{\infty}$-algebras. Crucially, the recursive definition of the minimal model brackets via (2.17) is identical to the recursive formula (4.7) for $\Phi(x)$ originally derived by the usual Legendre transform of formal power series (relating the 1PI functional $\Gamma$ to the generating functional $W$ of connected correlators), as applied to the asymptotic source $J_{\varphi}(x)$ sourcing incoming/outgoing 1-particle states. The cyclic $L_{\infty}$-algebra of the S-matrix this procedure yields is defined by the hamitonian $\Theta[\varphi]$ which is the S-matrix generating functional $\mathcal{A}[\varphi]$ (4.11) up to a sign, with cyclic inner product the restriction of (4.28) to the space of asymptotic 1-particle states.

\section{Discussion}

We argued that the most basic objects one usually cares about in quantum field theory - the S-matrix, and vacuum correlators - have a not-widely-appreciated $L_{\infty}$-algebraic structure. Moreover, we claim this structure is natural:

- the $L_{\infty}$ Jacobi identities are the non-anomalous Slavnov-Taylor identities;

- the $L_{\infty}$-algebra of the S-matrix is obtained from the $L_{\infty}$-algebra of correlators by a canonical construction, that is the minimal model theorem;

- Maurer-Cartan elements (2.12) are the extrema of the 1PI functional $\Gamma$, corresponding to the moduli space of vacua à la Coleman-Weinberg [19].

The facts involving the $L_{\infty}$-algebra of correlators alone follow by trivial observations from Zinn-Justin's 1974 work [4]; we have only given a dictionary that translates from his "antifield" or BV language to $L_{\infty}$ language. However, the relation to the S-matrix (outside of string field theory $[20,21,23,24,55]$ and the more recent independently-derived results of [25] for tree-level gravity and Yang-Mills) is new. 
An orthogonal but not incompatible interpretation of our results is that $L_{\infty}$-algebraic structures do not characterise closed string field theory (where they were originally discovered [10]), but are instead immanent to quantum field theory with a choice of vacuum. Supporting this is the last claim above: in the context of the philosophy which associates an $L_{\infty}$-algebra to a deformation problem [17], we see from the Coleman-Weinberg [19] calculation that the Maurer-Cartan moduli space (2.12) of the $L_{\infty}$-algebra of correlators corresponds to the vacuum moduli space.

The validity of our results hinges largely on

1. having a well-defined 1PI effective action $\Gamma$, known either exactly, or to some order in $\hbar$, satisfying the Zinn-Justin equation $(\Gamma, \Gamma)=0$;

2. (for the Minkowski space S-matrix:) having a mass gap, so the LSZ formula and Källén-Lehmann spectral representation [69, 70] are valid;

3. (also for the S-matrix:) there being no bound states.

Requirement 3. could conceivably be relaxed in general: if a bound state appearing in the S-matrix is created by a composite operator $\mathcal{O}[\phi](x)$ (where $x$ here is some sort of collective coordinate), one could source it using a current $J_{\mathcal{O}}(x)$ in the path integral to arrive at a generating functional $Z\left[J, J_{\mathcal{O}}, \stackrel{\star}{\Phi}\right]$, then take a logarithm and do a Legendre transform on both $J$ and $J_{\mathcal{O}}$ to try to derive a Zinn-Justin equation for a new kind of 1PI functional. This direction has been pursued in [76], including a Zinn-Justin equation for what is called a "master functional" therein.

With regard to requirement 1 . we emphasise that it is not necessary that $\Gamma$ be known exactly; our arguments work if for instance we have an expansion

$$
\Gamma=\Gamma_{0}+\hbar \Gamma_{1}+\cdots+\hbar^{n} \Gamma_{n}+O\left(\hbar^{n+1}\right)
$$

and we do not care to calculate the higher corrections. Here we only need to replace the field $\mathbb{R}$ or $\mathbb{C}$ of scalars of the $L_{\infty}$ algebra with the ring $\mathbb{R}[\hbar] /\left\{\hbar^{n+1}\right\}$ (where we quotiented formal power series in $\hbar$ by the ideal generated by $\hbar^{n+1}=0$ ). This produces a large class of examples, the most accessible of which are tree-level theories, where $\Gamma$ reduces to the classical action by the usual stationary-phase argument.

Relatedly, we have not considered questions of renormalisation. We simply assume $\Gamma$ has been defined at some mass scale $\mu^{2}$ so condition 1 . is satisfied. This does lead to a priori inequivalent $L_{\infty}$-algebras for each $\mu^{2}$. We prefer to resolve this in the future. One might expect to make contact with the recent treatment of renormalisation in the BV formalism [77] which is however based on the quantum master action $S$, not the 1PI functional $\Gamma$ (linked by (3.2), (3.7), and (3.8)).

As a mathematical aside we mention that the above relation between $S$ and $\Gamma$ appears to associate to any loop $L_{\infty}$-algebra [63] (say over $\mathbb{R}$ ) an ordinary $L_{\infty}$-algebra over $\mathbb{R}[\hbar]$ through a "perturbative path integral" expression. The Jacobi identities on either side are then related by the obvious generalisation of (3.8). The calculations showing the correspondence between the respective cohomologies (see e.g. [57] sections 8.4, 8.5) should 
be rigorous given that perturbative path integrals can be formalised, as in e.g. [78]. This appears to lead to a compact reformulation of the notion of loop $L_{\infty}$-algebra.

Requirement 2. - relevant for the S-matrix on Minkowski space - is the hardest to satisfy. The LSZ formula and the Källén-Lehmann spectral representation fail in the more interesting context of gauge theories: the former because gauge theories usually have massless degrees of freedom (i.e. no mass gap) which contradicts the assumption of free asymptotic 1-particle states, and the latter because the space of states is not a Hilbert space when ghosts are present (they have negative norm). Of these, the failure of the LSZ formula is the more serious one. Another way to phrase it is that infrared divergences obstruct the construction of an S-matrix. Of course, at tree-level there are no divergences and the S-matrix can indeed be obtained according to (4.11).

We now suggest applications and generalisations. One immediate application is to tree-level theories, which already generalises the aforementioned known results. In the paper [1] (which appeared at the same time as version 1 of this paper) this is pursued with the remarkable result that the minimal model theorem leads to practical recursion relations for scattering amplitudes for any tree-level theory. In our language this can be understood as follows: from (2.21) we see that an $n$-point amplitude is built off the quantities $f_{n b_{1} b_{2} \ldots b_{n}}^{a}$ calculated from (2.17). These depend only on the $m \leq(n-1)$-point amplitudes as well as the $n$-ary and lower structure constants of the $L_{\infty}$-algebra of correlators; at tree level, the latter are essentially the structure constants of the original lagrangian and are therefore known. In fact the $f_{n b_{1} b_{2} \ldots b_{n}}^{a}$ are almost the same as the Berends-Giele currents [51] as might be guessed from the fact they have the same index structure, involving $n$ on-shell legs and a single off-shell leg. The recursion (2.17) is then effectively identical to Berends-Giele recursion. While the recursion (2.17) also exists at loop level, it is less practical because the $L_{\infty}$-structure constants $C_{b_{1} \ldots b_{n}}^{a}$ it involves are then the correlators of the theory and are not known a priori.

Consider furthermore the problem of $S$-matrix equivalence i.e. of finding which field redefinitions lead to equivalent S-matrices [79-81]. Clearly the necessary and sufficient condition is that a field redefinition induces a quasi-isomorphism of the corresponding $L_{\infty^{-}}$ algebras of 1PI correlators. Checking this is not entirely straightforward, but probably easier than recalculating the S-matrix. For linear field redefinitions this is easy, however: consider e.g. acting with a Poincaré transformation on $\phi(x)$ of the last section. Since the result is linear in $\phi(x)$ we find $\Phi(x)$ transforms the same way (given that the vacuum is Poincaré invariant). This is trivially an $L_{\infty}$-isomorphism of cyclic $L_{\infty}$-algebras, showing Poincaré invariance of the S-matrix.

The $L_{\infty}$-algebraic perspective is well-suited to problems involving determining deformations of QFTs (or, rather, their correlation functions). Given our results, we see that the deformation problem is controlled in general by cyclic $L_{\infty}$-algebra cohomology [82], which can be reduced to the cohomology of the cyclic minimal model (as is obvious from (2.29)), i.e. the S-matrix. In fact applications vaguely along those lines have already appeared in the form of $[83,84]$ wherein non-commutative deformations of Chern-Simons and Yang-Mills were determined through an " $L_{\infty}$-bootstrap". 
Finally, consider the problem of extending our results to conformal field theories. As explained above, the construction in this paper does not directly apply ${ }^{6}$ so it is unclear what a "minimal model" of a CFT in the sense of the minimal model theorem should correspond to (again, this is a different notion than that of a minimal model CFT). A related curiocity is that all 2-point functions of any CFT are fixed by conformal symmetry. Something similar happens to the S-matrix functional: $1 \rightarrow 1$ scattering is trivial, so the quadratic term in any S-matrix functional is universal, as we already pointed out in the previous section. This suggests we could discard the 2-point functions in a CFT (since there is effectively no information therein) and see if $n$-point CFT correlators for $n \geq 3$ are the minimal model of a different theory. In fact, this seems to be realised by AdS/CFT, at least in the following simple example: taking scalar field correlators for simplicity, the dictionary of $[85,86]$ states that CFT correlators (given by a generating functional $Z_{\mathrm{CFT}}\left[\phi_{0}\right]$ ) at a large $N$ limit are well-approximated by the (renormalised) on-shell bulk Euclidean AdS action $I_{\mathrm{AdS}}[\phi]$ where $\phi_{0}$ is the boundary value of the on-shell bulk scalar field $\phi$ :

$$
Z_{\mathrm{CFT}}\left[\phi_{0}\right]=\exp \left(-I_{\mathrm{AdS}}[\phi]\right), \quad \phi \text { solves } \frac{\delta I_{\mathrm{AdS}}[\phi]}{\delta \phi}=0,\left.\quad \phi\right|_{\partial \mathrm{AdS}}=\phi_{0} .
$$

The analogy to the Jevicki-Lee S-matrix prescription is obvious, modulo the need to discard the 2-point contribution on either side.

\section{Acknowledgments}

It is a pleasure to acknowledge discussions and helpful feedback from Tim Adamo, Alec Barns-Graham, Panos Betzios, José Eliel Camargo-Molina, Joe Keir, Victor Lekeu, Iva Lovrekovic, Olga Papadoulaki, Matt Roberts, David Tennyson, and Dan Thomson. I also appreciate the clarifications of Tommaso Macrelli, Christian Sämann, and Martin Wolf regarding their work [1]. Special thanks goes to Jim Stasheff for encouragement and for pointing out certain references I missed.

I am supported by the EPSRC programme grant "New Geometric Structures from String Theory" (EP/K034456/1).

\section{A Cyclic Hodge-Kodaira decompositions}

We prove (2.14) and the claims around it, for finite $\operatorname{dim} \mathcal{V}$. Identity (2.6) for a cyclic inner product $\kappa_{a b}$ implies in particular for the unary bracket $K\left(T_{a}\right)=C_{a}^{b} T_{b}$

$$
\kappa_{a c} C_{b}^{c}=(-1)^{a b} \kappa_{b c} C_{a}^{c} \Longrightarrow \kappa\left(v_{1}, K v_{2}\right)=(-1)^{\left(\operatorname{deg} v_{1}\right)\left(\operatorname{deg} v_{2}\right)} \kappa\left(v_{2}, K v_{1}\right)
$$

whence

$$
(\operatorname{im} K)^{\perp}=\operatorname{ker} K \Longrightarrow(\operatorname{ker} K)^{\perp}=\operatorname{im} K
$$

(we need finite dimensionality for the last implication). Therefore $\kappa$ descends to a nondegenerate bilinear form on the cohomology $\operatorname{ker} K / \operatorname{im} K$ of $K$.

\footnotetext{
${ }^{6}$ As there is no mass gap or obvious notion of free asymptotic states to scatter.
} 
Now select a subspace $P$ of cohomology representatives in ker $K$ so that

$$
\text { ker } K=P \oplus \operatorname{im} K \text {. }
$$

We see that $\left.\kappa\right|_{P \times P}$ is non-degenerate, implying

$$
\mathcal{V}=P \oplus P^{\perp}
$$

where we stress $P \cap P^{\perp}=\{0\}$ and $\kappa$ is non-degenerate on both $P$ and $P^{\perp}$.

Since $\operatorname{im} K \subset P^{\perp}$ and $\operatorname{im} K \subset(\operatorname{im} K)^{\perp}$ it follows that $\kappa$ restricts to zero on im $K$ in $P^{\perp}$. For any complementary subspace $L$ so $P^{\perp}=\operatorname{im} K \oplus L$ is a direct sum, $K$ can be restricted to a map $\left.K\right|_{L}: L \rightarrow \operatorname{im} K$ where it is invertible, and $L \cong \operatorname{im~} K$ as vector spaces. Call the inverse $G: \operatorname{im} K \rightarrow L$ (which is necessarily a degree -1 linear map given $\operatorname{deg} K=+1)$ and extend it to a map $\mathcal{V} \rightarrow L$ by setting $G(v)=0 \quad \forall v \in P \oplus L \subset \mathcal{V}$. This in particular implies

$$
G^{2}=0
$$

and we get the identities

$$
(K G)^{2}=K G, \quad(G K)^{2}=G K \Longrightarrow G K G=G, \quad K G K=K
$$

Since $L$ and im $K$ are both $\kappa$-perpendicular to $P$, if $P$ denotes the projector onto $P$ we also get $K P=P K=K G=G K=0$.

$K G$ and $G K$ are projectors onto im $K$ and $L$ respectively, but not $\kappa$-orthogonal ones since im $K$ and $L$ can never be chosen to be orthogonal. However, we can select $L$ such that $\left.\kappa\right|_{L \times L}=0$ i.e. $\mathrm{im}(G K)$ is $\kappa$-null (easy to construct in finite dimensions). When this is the case, we obtain the identity

$$
\kappa_{a c} G_{b}^{c}=-(-1)^{a b} \kappa_{b c} G_{a}^{c} .
$$

To summarise: a "propagator" $G: \mathcal{V} \rightarrow \mathcal{V}$ of degree -1 compatible with $\kappa$ (A.7) exists and depends on a choice of cohomology representatives $P$ and a choice of "co-exact" elements $L \subset P^{\perp}$. This is true in finite dimensions. For a Hilbert space if we assume that $(\operatorname{im} K)$ and (ker $K)$ are both closed the argument goes through except for the point that we can always find an $L$ such that $\left.\kappa\right|_{L \times L}=0$.

\section{B Path integral derivation of the Zinn-Justin equation from the quantum master equation}

Using the definitions in the main text, since we only ever use left derivatives,

$$
(\Gamma, \Gamma)=2 \int d x(-1)^{\Phi} \frac{\delta \Gamma}{\delta \Phi(x)} \frac{\delta \Gamma}{\delta \Phi^{\star}(x)}=2 \int d x J(x) \frac{\delta \Gamma}{\delta \Phi^{\star}(x)} .
$$


We need to calculate $\delta \Gamma / \delta \stackrel{\star}{\Phi}(x)$ in terms of $Z[J, \stackrel{\star}{\Phi}]$. This is in fact proportional to $-i \hbar \delta \log Z[J, \stackrel{\star}{\Phi}] /\left.\delta \stackrel{\star}{\Phi}(x)\right|_{J=J[\Phi, \stackrel{\star}{\Phi}]}$ by the following short calculation: from the Legendre transform (3.7),

$$
\begin{aligned}
\frac{\delta \Gamma}{\delta \stackrel{\star}{\Phi}(x)} & =-i \hbar\left(\frac{\delta}{\delta \stackrel{\star}{\Phi}(x)} \log Z[J[\Phi, \stackrel{\star}{\Phi}], \stackrel{\star}{\Phi}]-\int d y \frac{\delta J(y)}{\delta \stackrel{\star}{\Phi}(x)} \Phi(y)\right) \\
& =-i \hbar\left(\left.\frac{\delta \log Z}{\delta \stackrel{\star}{\Phi}(x)}\right|_{J=J[\Phi, \stackrel{\star}{\Phi}]}+\int d y \frac{\delta J(y)}{\delta \frac{\star}{\Phi}(x)} \frac{\delta \log Z}{\delta J(y)}-\frac{\delta J(y)}{\delta^{\stackrel{\star}{\Phi}(x)}} \Phi(y)\right)
\end{aligned}
$$

so the last two terms cancel by (3.6). We used the chain rule for left derivatives $d f=d w^{a}\left(\partial z^{b} / \partial w^{a}\right)\left(\partial f / \partial z^{b}\right)$ (the order is important when fermion variables are involved).

Therefore

$$
(\Gamma, \Gamma)=2 \int d x \frac{1}{Z[J, \stackrel{\star}{\Phi}]} \int \mathcal{D} \phi J(x) e^{\int J \phi} \frac{\delta}{\delta \stackrel{\star}{\Phi}(x)} e^{i S / \hbar}
$$

where $J=J[\Phi, \stackrel{\star}{\Phi}]$ and $S=S[\phi, \stackrel{\star}{\Phi}+\delta \Psi / \delta \phi]$ as in section 3. Using the trick $J(x) \exp \left(\int J \phi\right)=(-1)^{\phi} \delta \exp \left(\int J \phi\right) / \delta \phi(x)$, we can integrate by parts inside the path integral to move $\delta / \delta \phi(x)$ to $\exp (i S / \hbar)$ (at the expense of a total derivative term, which we discard). The end result is

$$
(\Gamma, \Gamma)=-2 \int d x \frac{1}{Z[J, \stackrel{\star}{\Phi}]} \int \mathcal{D} \phi e^{\int J \phi} \Delta e^{i S / \hbar}
$$

(where the $x$ integral smears $\Delta=(-1)^{\phi} \delta^{2} / \delta \phi(x) \delta \hat{\phi}(x)$ in $x$ ) which leads to the result in the main text.

Open Access. This article is distributed under the terms of the Creative Commons Attribution License (CC-BY 4.0), which permits any use, distribution and reproduction in any medium, provided the original author(s) and source are credited.

\section{References}

[1] T. Macrelli, C. Sämann and M. Wolf, Scattering Amplitude Recursion Relations in BV Quantisable Theories, arXiv:1903.05713 [INSPIRE].

[2] H. Lehmann, K. Symanzik and W. Zimmermann, On the formulation of quantized field theories, Nuovo Cim. 1 (1955) 205 [INSPIRE].

[3] C. Itzykson and J.B. Zuber, Quantum Field Theory, International Series In Pure and Applied Physics, McGraw-Hill, New York (1980) [INSPIRE].

[4] J. Zinn-Justin, Renormalization of Gauge Theories, Lect. Notes Phys. 37 (1975) 1 [inSPIRE].

[5] I.A. Batalin and G.A. Vilkovisky, Quantization of Gauge Theories with Linearly Dependent Generators, Phys. Rev. D 28 (1983) 2567 [Erratum ibid. D 30 (1984) 508] [InSPIRE].

[6] I.A. Batalin and G.A. Vilkovisky, Existence Theorem for Gauge Algebra, J. Math. Phys. 26 (1985) 172 [INSPIRE]. 
[7] I.A. Batalin and G.A. Vilkovisky, Closure of the Gauge Algebra, Generalized Lie Equations and Feynman Rules, Nucl. Phys. B 234 (1984) 106 [InSPIRE].

[8] I.A. Batalin and G.A. Vilkovisky, Gauge Algebra and Quantization, Phys. Lett. 102B (1981) 27 [INSPIRE].

[9] I.A. Batalin and G.A. Vilkovisky, Relativistic S Matrix of Dynamical Systems with Boson and Fermion Constraints, Phys. Lett. 69B (1977) 309 [INSPIRE].

[10] B. Zwiebach, Closed string field theory: Quantum action and the B-V master equation, Nucl. Phys. B 390 (1993) 33 [hep-th/9206084] [INSPIRE].

[11] T. Lada and J. Stasheff, Introduction to SH Lie algebras for physicists, Int. J. Theor. Phys. 32 (1993) 1087 [hep-th/9209099] [INSPIRE].

[12] R. D'Auria and P. Fré, Geometric Supergravity in d =11 and Its Hidden Supergroup, Nucl. Phys. B 201 (1982) 101 [Erratum ibid. B 206 (1982) 496] [InSPIRE].

[13] R. D'Auria, P. Fré and T. Regge, Graded Lie Algebra Cohomology and Supergravity, Riv. Nuovo Cim. 3N12 (1980) 1 [INSPIRE].

[14] L. Castellani, P. Fré, F. Giani, K. Pilch and P. van Nieuwenhuizen, Gauging of $d=11$ Supergravity?, Annals Phys. 146 (1983) 35 [InSPIRE].

[15] H. Sati, U. Schreiber and J. Stasheff, $L_{\infty}$ algebra connections and applications to String- and Chern-Simons n-transport, in Quantum Field Theory, B. Fauser, J. Tolksdorf and E. Zeidler eds., Birkhauser (2009) [arXiv:0801.3480] [INSPIRE].

[16] F.A. Berends, G.J.H. Burgers and H. van Dam, On the Theoretical Problems in Constructing Interactions Involving Higher Spin Massless Particles, Nucl. Phys. B 260 (1985) 295 [INSPIRE].

[17] M. Schlessinger and J. Stasheff, Deformation theory and rational homotopy type, arXiv: 1211.1647.

[18] A. Sen, Equations of Motion in Nonpolynomial Closed String Field Theory and Conformal Invariance of Two-dimensional Field Theories, Phys. Lett. B 241 (1990) 350 [InSPIRE].

[19] S.R. Coleman and E.J. Weinberg, Radiative Corrections as the Origin of Spontaneous Symmetry Breaking, Phys. Rev. D 7 (1973) 1888 [INSPIRE].

[20] E. Witten and B. Zwiebach, Algebraic structures and differential geometry in 2-D string theory, Nucl. Phys. B 377 (1992) 55 [hep-th/9201056] [InSPIRE].

[21] E.P. Verlinde, The Master equation of 2-D string theory, Nucl. Phys. B 381 (1992) 141 [hep-th/9202021] [INSPIRE].

[22] H. Kajiura, Noncommutative homotopy algebras associated with open strings, Rev. Math. Phys. 19 (2007) 1 [math/0306332] [INSPIRE].

[23] K. Münster and I. Sachs, Homotopy Classification of Bosonic String Field Theory, Commun. Math. Phys. 330 (2014) 1227 [arXiv:1208.5626] [INSPIRE].

[24] S. Konopka, The S-matrix of superstring field theory, JHEP 11 (2015) 187 [arXiv: 1507.08250] [INSPIRE].

[25] A. Nützi and M. Reiterer, Scattering amplitudes in YM and GR as minimal model brackets and their recursive characterization, arXiv:1812.06454 [INSPIRE]. 
[26] A. Jevicki and C.-k. Lee, The S Matrix Generating Functional and Effective Action, Phys. Rev. D 37 (1988) 1485 [INSPIRE].

[27] O. Hohm and B. Zwiebach, $L_{\infty}$ Algebras and Field Theory, Fortsch. Phys. 65 (2017) 1700014 [arXiv: 1701.08824] [INSPIRE].

[28] B. Jurčo, L. Raspollini, C. Sämann and M. Wolf, $L_{\infty}$-Algebras of Classical Field Theories and the Batalin-Vilkovisky Formalism, arXiv:1809.09899 [INSPIRE].

[29] P. Ritter and C. Sämann, $L_{\infty}$-Algebra Models and Higher Chern-Simons Theories, Rev. Math. Phys. 28 (2016) 1650021 [arXiv:1511.08201] [INSPIRE].

[30] D. Fiorenza, C.L. Rogers and U. Schreiber, A Higher Chern-Weil derivation of AKSZ o-models, Int. J. Geom. Meth. Mod. Phys. 10 (2013) 1250078 [arXiv:1108.4378] [inSPIRE].

[31] A. Kotov and T. Strobl, Characteristic classes associated to Q-bundles, Int. J. Geom. Meth. Mod. Phys. 12 (2014) 1550006 [arXiv:0711.4106] [InSPIRE].

[32] O. Hohm, V. Kupriyanov, D. Lüst and M. Traube, Constructions of $L_{\infty}$ algebras and their field theory realizations, Adv. Math. Phys. 2018 (2018) 9282905 [arXiv:1709.10004] [INSPIRE].

[33] R. Blumenhagen, M. Fuchs and M. Traube, $\mathcal{W}$ algebras are $L_{\infty}$ algebras, JHEP 07 (2017) 060 [arXiv: 1705. 00736] [INSPIRE].

[34] M. Cederwall and J. Palmkvist, $L_{\infty}$ algebras for extended geometry from Borcherds superalgebras, Commun. Math. Phys. 369 (2019) 721 [arXiv: 1804.04377] [INSPIRE].

[35] Y. Cagnacci, T. Codina and D. Marques, $L_{\infty}$ algebras and Tensor Hierarchies in Exceptional Field Theory and Gauged Supergravity, JHEP 01 (2019) 117 [arXiv:1807.06028] [INSPIRE].

[36] S. Lavau, H. Samtleben and T. Strobl, Hidden Q-structure and Lie 3-algebra for non-abelian superconformal models in six dimensions, J. Geom. Phys. 86 (2014) 497 [arXiv:1403.7114] [INSPIRE].

[37] S. Lavau, Tensor hierarchies and Leibniz algebras, J. Geom. Phys. 144 (2019) 147 [arXiv: 1708.07068] [INSPIRE].

[38] A.S. Arvanitakis, Brane Wess-Zumino terms from AKSZ and exceptional generalised geometry as an $L_{\infty}$-algebroid, arXiv: 1804.07303 [INSPIRE].

[39] M. Alexandrov, A. Schwarz, O. Zaboronsky and M. Kontsevich, The Geometry of the master equation and topological quantum field theory, Int. J. Mod. Phys. A 12 (1997) 1405 [hep-th/9502010] [INSPIRE].

[40] J.M.L. Fisch and M. Henneaux, Homological Perturbation Theory and the Algebraic Structure of the Antifield-Antibracket Formalism for Gauge Theories, Commun. Math. Phys. 128 (1990) 627 [INSPIRE].

[41] G. Barnich, R. Fulp, T. Lada and J. Stasheff, The sh Lie structure of Poisson brackets in field theory, Commun. Math. Phys. 191 (1998) 585 [hep-th/9702176] [INSPIRE].

[42] R. Fulp, T. Lada and J. Stasheff, sh-Lie algebras induced by gauge transformations, Commun. Math. Phys. 231 (2002) 25 [inSPIRE].

[43] M. Movshev and A.S. Schwarz, On maximally supersymmetric Yang-Mills theories, Nucl. Phys. B 681 (2004) 324 [hep-th/0311132] [INSPIRE].

[44] M. Movshev and A.S. Schwarz, Algebraic structure of Yang-Mills theory, Prog. Math. 244 (2006) 473 [hep-th/0404183] [inSPIRE]. 
[45] A.M. Zeitlin, Homotopy Lie Superalgebra in Yang-Mills Theory, JHEP 09 (2007) 068 [arXiv:0708.1773] [INSPIRE].

[46] A.M. Zeitlin, Formal Maurer-Cartan Structures: From CFT to Classical Field Equations, JHEP 12 (2007) 098 [arXiv:0708.0955] [INSPIRE].

[47] A.M. Zeitlin, Batalin-Vilkovisky Yang-Mills theory as a homotopy Chern-Simons theory via string field theory, Int. J. Mod. Phys. A 24 (2009) 1309 [arXiv:0709.1411] [InSPIRE].

[48] A.M. Zeitlin, String field theory-inspired algebraic structures in gauge theories, J. Math. Phys. 50 (2009) 063501 [arXiv: 0711.3843] [INSPIRE].

[49] A.M. Zeitlin, Conformal Field Theory and Algebraic Structure of Gauge Theory, JHEP 03 (2010) 056 [arXiv: 0812.1840] [INSPIRE].

[50] M. Roček and A.M. Zeitlin, Homotopy algebras of differential (super)forms in three and four dimensions, Lett. Math. Phys. 108 (2018) 2669 [arXiv:1702.03565] [INSPIRE].

[51] F.A. Berends and W.T. Giele, Recursive Calculations for Processes with n Gluons, Nucl. Phys. B 306 (1988) 759 [INSPIRE].

[52] M. Kontsevich, Deformation quantization of Poisson manifolds. 1., Lett. Math. Phys. 66 (2003) 157 [q-alg/9709040] [INSPIRE].

[53] C. Braun and A. Lazarev, Unimodular homotopy algebras and Chern-Simons theory, J. Pure Appl. Algebra 219 (2015) 5158 [arXiv: 1309.3219] [InSPIRE].

[54] T.V. Kadeishvili, On the homology theory of fibre spaces, Russ. Math. Surv. 35 (1980) 231.

[55] H. Kajiura, Homotopy algebra morphism and geometry of classical string field theory, Nucl. Phys. B 630 (2002) 361 [hep-th/0112228] [INSPIRE].

[56] J.-L. Loday and B. Vallette, Algebraic operads, vol. 346, Springer Science \& Business Media (2012).

[57] J. Gomis, J. Paris and S. Samuel, Antibracket, antifields and gauge theory quantization, Phys. Rept. 259 (1995) 1 [hep-th/9412228] [INSPIRE].

[58] A.S. Arvanitakis, Chiral strings, topological branes and a generalised Weyl-invariance, Int. J. Mod. Phys. A 34 (2019) 1950031 [arXiv:1705.03516] [InSPIRE].

[59] M. Henneaux and C. Teitelboim, Quantization of gauge systems, Princeton University Press (1992).

[60] D.M. Jackson, A. Kempf and A.H. Morales, A robust generalization of the Legendre transform for QFT, J. Phys. A 50 (2017) 225201 [arXiv: 1612.00462] [INSPIRE].

[61] D. Anselmi, Removal of divergences with the Batalin-Vilkovisky formalism, Class. Quant. Grav. 11 (1994) 2181 [INSPIRE].

[62] G. Barnich, Classical and quantum aspects of the extended antifield formalism, Ann. U. Craiova Phys. 10 (2000) 1 [hep-th/0011120] [INSPIRE].

[63] M. Markl, Loop homotopy algebras in closed string field theory, Commun. Math. Phys. 221 (2001) 367 [hep-th/9711045] [INSPIRE].

[64] R. Fukuda, M. Komachiya and M. Ukita, On-shell Expansion of the Effective Action: $S$ Matrix and the Ambiguity-Free Stability Criterion, Phys. Rev. D 38 (1988) 3747 [InSPIRE].

[65] C.-j. Kim and V.P. Nair, Recursion rules for scattering amplitudes in nonAbelian gauge theories, Phys. Rev. D 55 (1997) 3851 [hep-th/9608156] [INSPIRE]. 
[66] V.P. Nair, Quantum field theory: A modern perspective. Springer (2005).

[67] W. Siegel, Fields, hep-th/9912205 [inSPIRE].

[68] I.Y. Arefeva, L.D. Faddeev and A.A. Slavnov, Generating Functional for the s Matrix in Gauge Theories, Theor. Math. Phys. 21 (1975) 1165 [InSPIRE].

[69] G. Kallen, On the definition of the Renormalization Constants in Quantum Electrodynamics, Helv. Phys. Acta 25 (1952) 417 [inSPIRE].

[70] H. Lehmann, On the Properties of propagation functions and renormalization contants of quantized fields, Nuovo Cim. 11 (1954) 342 [INSPIRE].

[71] M. Reed and B. Simon, Methods of modern mathematical physics, vol. III: Scattering theory, Academic Press, New York, San Francisoco, London (1979).

[72] E. Zeidler ed., Quantum field theory. I: Basics in mathematics and physics. A bridge between mathematicians and physicists, Springer (2006).

[73] T. Adamo, S. Nakach and A.A. Tseytlin, Scattering of conformal higher spin fields, JHEP 07 (2018) 016 [arXiv: 1805.00394] [INSPIRE].

[74] T. Adamo, E. Casali, L. Mason and S. Nekovar, Scattering on plane waves and the double copy, Class. Quant. Grav. 35 (2018) 015004 [arXiv:1706.08925] [InSPIRE].

[75] K.J. Costello, Renormalisation and the Batalin-Vilkovisky formalism, arXiv:0706.1533 [INSPIRE].

[76] D. Anselmi, Master Functional And Proper Formalism For Quantum Gauge Field Theory, Eur. Phys. J. C 73 (2013) 2363 [arXiv: 1205.3862] [INSPIRE].

[77] K. Costello, Renormalization and effective field theory, No. 170, American Mathematical Soc. (2011).

[78] C. Braun and J. Maunder, Minimal models of quantum homotopy Lie algebras via the BV-formalism, J. Math. Phys. 59 (2018) 063512 [arXiv:1703.00082] [INSPIRE].

[79] J.S.R. Chisholm, Change of variables in quantum field theories, Nucl. Phys. 26 (1961) 469.

[80] S. Kamefuchi, L. O'Raifeartaigh and A. Salam, Change of variables and equivalence theorems in quantum field theories, Nucl. Phys. 28 (1961) 529.

[81] R.E. Kallosh and I.V. Tyutin, The Equivalence theorem and gauge invariance in renormalizable theories, Yad. Fiz. 17 (1973) 190 [InSPIRE].

[82] M. Penkava and A.S. Schwarz, $A_{\infty}$ algebras and the cohomology of moduli spaces, hep-th/9408064 [INSPIRE].

[83] R. Blumenhagen, I. Brunner, V. Kupriyanov and D. Lüst, Bootstrapping non-commutative gauge theories from $L_{\infty}$ algebras, JHEP 05 (2018) 097 [arXiv: 1803.00732] [INSPIRE].

[84] R. Blumenhagen, M. Brinkmann, V. Kupriyanov and M. Traube, On the Uniqueness of $L_{\infty}$ bootstrap: Quasi-isomorphisms are Seiberg-Witten Maps, J. Math. Phys. 59 (2018) 123505 [arXiv: 1806.10314] [INSPIRE].

[85] E. Witten, Anti-de Sitter space and holography, Adv. Theor. Math. Phys. 2 (1998) 253 [hep-th/9802150] [INSPIRE].

[86] S.S. Gubser, I.R. Klebanov and A.M. Polyakov, Gauge theory correlators from noncritical string theory, Phys. Lett. B 428 (1998) 105 [hep-th/9802109] [INSPIRE]. 